\title{
Comparison of Cerebral Blood Flow Patterns in Patients with Phantom Bite Syndrome with Their Corresponding Clinical Features
}

This article was published in the following Dove Press journal: Neuropsychiatric Disease and Treatment

Yojiro Umezaki, (D) ' Motoko Watanabe, ${ }^{2}$ Yukiko Shinohara, (ID) ${ }^{2}$ Shiori Sugawara, (iD) ${ }^{2}$ Kaoru Kawasaki, (D) ${ }^{2}$ Trang TH Tu, (D) ${ }^{2}$ Takeshi Watanabe, (iD) ${ }^{2}$ Takayuki Suga, (iD) ${ }^{2}$ Anna Miura, (D) ${ }^{2}$ Miho Takenoshita, ${ }^{2}$ Yusuke Sato, (ID) ${ }^{3}$ Ichiro Minami, (iD) ${ }^{4}$ Jun Oyama, ${ }^{5}$ Akira Toriihara, (iD ${ }^{5}$ Tatsuya Yoshikawa, ${ }^{2}$ Toru Naito,' Haruhiko Motomura, ${ }^{2}$ Akira Toyofuku (iD) ${ }^{2}$

'Section of Geriatric Dentistry, Department of General Dentistry, Fukuoka Dental College, Fukuoka 8I40193, Japan; ${ }^{2}$ Department of Psychosomatic Dentistry, Graduate School of Medical and Dental Sciences, Tokyo Medical and Dental University, Tokyo I I 3-8548, Japan; ${ }^{3}$ Department of Gerontology and Gerodontology, Graduate School of Medical and Dental Sciences, Tokyo Medical and Dental University, Tokyo I I3-8548, Japan; ${ }^{4}$ Department of Removable Partial Prosthodontics, Graduate School of Medical and Dental Sciences, Tokyo Medical and Dental University, Tokyo I I3-8548, Japan; ${ }^{5}$ Department of Diagnostic Radiology and Nuclear Medicine, Graduate School of Medical and Dental Sciences, Tokyo Medical and Dental University, Tokyo I I3-8548, Japan

Correspondence: Akira Toyofuku Department of Psychosomatic Dentistry, Graduate School of Medical and Dental Sciences, Tokyo Medical and Dental University, I-5-45 Yushima, Bunkyo-Ku, Tokyo II 3-8548, Japan

$\mathrm{Tel} / \mathrm{Fax}+8 \mathrm{I}$ - 3-5803-5909 Email

toyoompm@tmd.ac.jp
Background: Phantom bite syndrome (PBS) is characterized by an uncomfortable sensation during occlusion without any evident abnormality. A recent case-control study with singlephoton emission computed tomography (SPECT) using ${ }^{99 \mathrm{~m}}$ Tc-ethyl cysteinate dimer could not find the specific features of regional cerebral blood flow (rCBF), which might be due to the heterogeneity of PBS. We analyzed the brain images of PBS corresponding to the clinical features by studying PBS subgroups.

Methods: This study contributes to elucidating the pathophysiology of PBS by evaluating regional brain perfusion on SPECT and its clinical features. We performed SPECT using ${ }^{99} \mathrm{~m}$ Tc-ethyl cysteinate dimer in 44 patients with PBS. The SPECT images were analyzed qualitatively and quantitatively.

Results: Asymmetrical rCBF patterns were detected, corresponding to symptom laterality. Patients with PBS with right-side symptoms showed right-side-predominant rCBF asymmetry in the parietal region and left-side-predominant $\mathrm{rCBF}$ asymmetry in the thalamus, and vice versa. Moreover, the analysis of the association between $\mathrm{rCBF}$ and patient behaviors revealed that patients who blamed their dentists for their symptoms tended to have a symmetrical $\mathrm{rCBF}$ pattern.

Conclusion: Patients with PBS showed blood flow imbalance in the thalamus and parietal region corresponding to symptom laterality. There are two types of symmetrical and asymmetrical rCBF patterns in the pathophysiology of PBS despite similar clinical manifestations.

Keywords: phantom bite syndrome, cerebral blood flow, single-photon emission computed tomography

\section{Background}

Phantom bite syndrome (PBS) is a term first described by Marbach $^{1-3}$ as the patients who stick to believing their dental occlusion to be abnormal, regardless of the occlusion itself. The complaints include "the bite is off," "the bite is sliding," "the bite is somewhat high/low," and so on. The belief of "wrong bite" in patients with PBS is unwavering, which prompts them to visit several doctors. Facile occlusal adjustment could offer temporary improvement, but eventually worsens the problems. Thereafter, patients with PBS report occlusal dysesthesia, ${ }^{4,5}$ indicating that this syndrome is not uncommon. On surveying reports on behaviors in PBS, many features were detected to be common. These patients are often preoccupied with their occlusion or teeth alignment, resulting in an obsession with 
dental treatment. Most of them have a history of unsuccessful dental treatments, and they blame the failure on the dentists' "unskilled" work. Medically unexplained symptoms, such as chronic back pain and body tilt, are often complaints associated with "wrong bites."

PBS was originally regarded as a psychogenic or psychiatric disorder such as paranoia, severe personality disorder, ${ }^{1,2}$ monosymptomatic hypochondriacal psychosis, and dysmorphophobia. ${ }^{3,6,7}$ However, recent studies have suggested central nervous system dysfunction in patients with PBS, partly based on the response to psychopharmacologic therapy ${ }^{4,8-10}$ and studies for other phantom sensations. ${ }^{11,12}$ Marbach $^{13}$ revised the interpretation of PBS to "altered central processing." We sustained this hypothesis in light of psychiatric comorbidities and psychopharmacological outcomes ${ }^{14}$ and the distribution of the side with occlusal discomfort. ${ }^{15}$ Moreover, we report a case of PBS in which symptoms improved with psychopharmacology along with a change in asymmetrical regional cerebral blood flow (rCBF) patterns, as evaluated by ${ }^{99 \mathrm{~m}}$ Tc-ethyl cysteinate dimer $\left({ }^{99 \mathrm{~m}} \mathrm{Tc}-\mathrm{ECD}\right)$ single-photon emission computed tomography (SPECT), ${ }^{16}$ which suggests the involvement of dysfunction in the central nervous system in patients with PBS.

rCBF patterns in patients with PBS were not significantly different from those in control participants of our recent case-control study as assessed using ${ }^{99 \mathrm{~m}} \mathrm{Tc}-\mathrm{ECD}$ SPECT. ${ }^{17}$ In this study, we speculated that the reason for this contradiction might suggest the heterogeneity of PBS. It may be possible that, from the very beginning, the scheme of comparison of patients with PBS with normal controls was inappropriate. Although our former study could not find unique rCBF patterns of all patients with PBS, we noticed that some clinical features could explain the variety of $\mathrm{rCBF}$ patterns. This could imply that the underlying pathophysiology of PBS in each individual patient comprises different proportions of dysfunctional central processing and other "psychogenic" factors. Since scrutinizing and assessing socio-psycho-behavioral factors is complicated and time-consuming, we focused on the relationship between $\mathrm{rCBF}$ patterns and objectifiable clinical features such as symptom laterality ${ }^{15}$ and behavioral features. In the present study, we hypothesized that any classification corresponding to clinical features could explain the diversity of rCBF patterns in PBS. To search for subgroups of PBS, we analyzed brain images of PBS corresponding to clinical features. This classification might promote individualized treatment of PBS.

\section{Methods}

\section{Study Design}

We analyzed the data from a recently published brain perfusion-based study for phantom bite syndrome. ${ }^{17}$ This was a case-control study to detect the pathophysiology of PBS using SPECT. Sixty-six subjects with PBS and 15 subjects without PBS were initially enrolled in the study. Subjects with and without PBS were recruited from the department of psychosomatic dentistry and prosthodontic dentistry, respectively. The diagnosis for PBS was based on a previous study. ${ }^{10}$ The exclusion criteria were severe psychiatric diseases, presence of organic problems in the brain as judged by a radiologist, cognitive impairment as judged by the Revised Hasegawa Dementia Scale (HDS-R) $(<25)$, abnormalities observed in the magnetic resonance imaging (MRI) of the brain except for a high signal intensity on the T2-weighted images that was not significant, and lefthanded patients defined by the Edinburgh inventory. We also excluded patients who had comorbid oral cenesthopathy, as the rCBF pattern in oral cenesthopathy ${ }^{18}$ could affect that of PBS. A total of 22 patients were excluded from the study, and 44 patients with PBS and 12 individuals without PBS were included.

In the present study, the detailed complaints of 44 patients with PBS were available: laterality of the symptoms (both sides, left, and right), presence or absence of sensation of biting discomfort, horizontal (bite is sliding, or slipping), and vertical (high or low) discrepancies. Three behavioral features assessed in these patients with regard to their dental visits (blaming dentists, sticking to occlusal treatment, and drug compliance) were also evaluated. This study was conducted with the approval of the Ethical Committee of Tokyo Medical and Dental University (No. 24,593,141).

\section{Brain Perfusion SPECT}

SPECT was performed at a fixed time for all subjects. The details of the SPECT protocol have been mentioned in our previous studies. ${ }^{17-19}$ Data acquisition began after the injection of a bolus of $600 \mathrm{MBq}$ of ${ }^{99 \mathrm{~m}} \mathrm{Tc}-\mathrm{ECD}$ through the right brachial vein with the patients' eyes closed. SPECT images were obtained using a rectangular, largefield, dual-head gamma camera (E.C. signature; Toshiba, Tokyo, Japan) equipped with fan-beam collimators after the passage from the heart to the brain for $100 \mathrm{~s}$ was monitored using the same gamma camera equipped with low-energy, high-resolution, and parallel-hole collimators. 


\section{Qualitative and Quantitative Analyses}

The evaluation of $\mathrm{rCBF}$ was the same as that in our previous study. ${ }^{17}$ For qualitative analysis, two nuclear medicine physicians qualitatively evaluated SPECT images of the participants after blinded group allocation. They visually judged whether rCBF asymmetry between the right and left sides of the brain perfusion existed in each of the five areas (frontal, temporal, parietal, lenticular nucleus, and thalamus). Consensus in imaging interpretation between the two nuclear medicine physicians was done for all participants. Subjects with an asymmetrical rCBF pattern in at least one region were allocated to the "asymmetry group." In contrast, those without asymmetrical rCBF patterns in any region were allocated to the "symmetry group"; among the 44 PBS cases, 17 were categorized into this group. SPECT images of patients in the asymmetry and symmetry groups are presented in Figure 1A and B, respectively.

To quantify CBF, the Patlak plot method was applied to the SPECT images to measure the mean CBF values. Subsequently, rCBF quantification was performed using a three-dimensional stereotactic region of interest template (3DSRT) program ${ }^{20,21}$ without correcting the partial-volume effect (PEV). 3DSRT is a fully automated rCBF quantification program that can be used to examine a total of $636 \mathrm{ROIs}$. These 636 ROIs are categorized into 12 brain segments on the 3DSRT template: callosomarginal, precentral, central, parietal, angular, temporal, posterior cerebral, pericallosal, lenticular, thalamic, hippocampal, and cerebellar segments. The blood flow to each ROI was quantified in $\mathrm{mL} / 100 \mathrm{~g} / \mathrm{min}$. The rCBF values obtained from the 3DSRT data were used in the quantitative analysis.

As an index of brain perfusion asymmetry, the $[R /(R+$ L)] ratio was calculated for the 12 brain segments in each participant, as follows:

$[\mathrm{R} /(\mathrm{R}+\mathrm{L})]=$ right $\mathrm{CBF} /$ sum of right and left $\mathrm{CBF}$ in each ROI.
In detail, $[\mathrm{R} /(\mathrm{R}+\mathrm{L})]$ ratio means $\mathrm{rCBF}$ values for the concerned segment on the right side $\times 100$ /sum of the rCBF values for the corresponding segments on the right and left sides. The mean $[\mathrm{R} /(\mathrm{R}+\mathrm{L})]$ ratios were calculated for each brain segment. The results are expressed as mean \pm standard deviation (SD).

\section{Statistical Analysis}

The data obtained were analyzed with the Kruskal-Wallis, Mann-Whitney U, Spearman correlation coefficient, and chi-square tests using the PASW software (IBM, Chicago, IL). $P$-values of $<0.05$ were considered to indicate statistical significance. Scheffe's test was performed to correct the multivariable comparison.

\section{Results}

\section{Symptoms of PBS in Patients}

Twenty-four patients ( 7 males and 17 females; mean age: $58.29 \pm 15.7$ years) complained of occlusal discomfort on both sides, and 11 (5 males and 6 females; mean age: $53.91 \pm 12.6$ years $)$ and 9 ( 1 male and 8 female; mean age: $61.44 \pm 10.2$ years) patients complained of left- or right-side symptoms, respectively. The Kruskal-Wallis test did not detect significant differences in age and sex between the three groups.

Complaints concerning horizontal bite discomfort such as "bite sliding" or "slipping" were seen in 20 patients (5 males and 15 females). Vertical bite discomfort such as "high or low bites" was seen in 18 patients ( 7 males and 11 females). Mean ages of the patients with horizontal and vertical complaints were $57.84 \pm 14.0$ years and $57.67 \pm 13.8$ years, respectively. No significant difference in age was detected between men and women in the horizontal bite discomfort group. However, in the vertical bite discomfort group, women were significantly older $(p=0.035)$, as evaluated using the Mann-Whitney $U$-test.
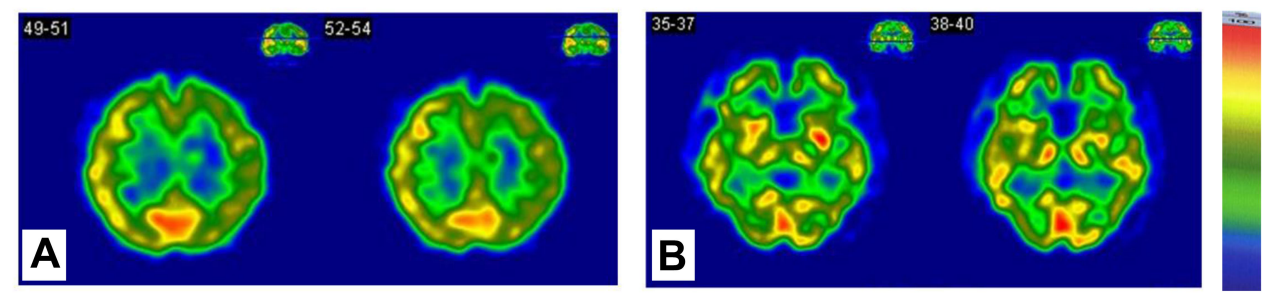

Figure I ${ }^{99 m} \mathrm{Tc}-\mathrm{ECD}$ single positron emission computed tomography images of patients with phantom bite syndrome. Patient (A) is a 67-year-old woman with right-side symptom. As compared to the values on the left side, the right frontal lobe shows higher perfusion values. Patient (B) is a 7I-year-old woman with bilateral symptom; no asymmetrical perfusion pattern is seen. 
Table I Correlation Between Patients' Clinical Features and Qualitative rCBF Asymmetry

\begin{tabular}{|c|c|c|c|}
\hline & & \multicolumn{2}{|l|}{ rCBF Pattern } \\
\hline & & “Asymmetry Group” & "Symmetry Group" \\
\hline \multicolumn{4}{|l|}{ Complaints } \\
\hline Symptom laterality & $\begin{array}{l}\text { Left } \\
\text { Both } \\
\text { Right }\end{array}$ & $\begin{array}{l}8 \\
13 \\
6\end{array}$ & $\begin{array}{l}3 \\
11 \\
3\end{array}$ \\
\hline Horizontal bite discomfort & $\begin{array}{l}\text { Yes } \\
\text { No }\end{array}$ & $\begin{array}{l}12 \\
15\end{array}$ & $\begin{array}{l}8 \\
9\end{array}$ \\
\hline Vertical bite discomfort & $\begin{array}{l}\text { Yes } \\
\text { No }\end{array}$ & $\begin{array}{l}11 \\
16\end{array}$ & $\begin{array}{l}7 \\
10\end{array}$ \\
\hline \multicolumn{4}{|l|}{ Behavioral features } \\
\hline Blaming dentists* & $\begin{array}{l}\text { Yes } \\
\text { No }\end{array}$ & $\begin{array}{l}6 \\
21\end{array}$ & $\begin{array}{l}10 \\
7\end{array}$ \\
\hline Sticking to occlusal treatment & $\begin{array}{l}\text { Yes } \\
\text { No }\end{array}$ & $\begin{array}{l}23 \\
4\end{array}$ & $\begin{array}{l}13 \\
4\end{array}$ \\
\hline Drug compliance & $\begin{array}{l}\text { Yes } \\
\text { No }\end{array}$ & $\begin{array}{l}17 \\
10\end{array}$ & $\begin{array}{l}8 \\
9\end{array}$ \\
\hline
\end{tabular}

Notes: * $p<0.05$ (chi-square test). "Asymmetry group": Patients with an asymmetrical rCBF pattern in at least one region. "Symmetry group": Patients with no asymmetrical $\mathrm{rCBF}$ pattern in any region on visual assessment.

Abbreviations: $\mathrm{rCBF}$, regional cerebral blood flow; PBS, phantom bite syndrome.

\section{Visual Qualitative Assessment of SPECT Images}

Table 1 shows the correlation between various clinical features and $\mathrm{rCBF}$ asymmetry using qualitative categorization of rCBF patterns. Asymmetrical rCBF was not significantly correlated with detailed complaints (laterality of oral symptoms, sensation of discomfort in the bite, whether vertical or horizontal). Among behavioral features (blaming dentists, sticking to dental treatment, and drug compliance), a significant difference was detected only between "blaming dentists" and asymmetry (chi-square test, $p=0.014$ ). Patients who were "blaming dentists" tended to have symmetrical rCBF.

\section{Quantitative Analysis of SPECT Images}

$\mathrm{R} /(\mathrm{R}+\mathrm{L})$ ratios were not significantly different between the presence and absence of vertical and horizontal bite discomfort (data not shown). In contrast, when patients with PBS were classified according to the symptomatic side (left-side symptoms, bilateral symptoms, and right-side symptoms), significant differences were detected in the precentral (leftside symptom: $49.95 \pm 0.45$, bilateral symptom: $50.43 \pm 0.66$, right-side symptom: $50.51 \pm 0.42 ; p=0.049$ ), parietal (left-side symptom: $49.69 \pm 0.73$, bilateral symptom: $50.24 \pm 0.75$, rightside symptom: $50.58 \pm 0.54 ; p=0.023$ ), and thalamic (left-side symptom: $50.84 \pm 1.55$, bilateral symptom: $50.19 \pm 1.31$, rightside symptom: $49.20 \pm 0.94 ; p=0.020$ ) regions with the Kruskal-Wallis test. Furthermore, the Mann-Whitney $U$-test corrected with Scheffe's test was used for all pairs in these three significant areas. In the parietal region, significantly lower $\mathrm{R} /(\mathrm{R}+\mathrm{L})$ ratios were observed in patients with left-sided symptoms and a higher ratio was observed in patients with right-sided symptoms $(p=0.029)$; however, the tendency was opposite in the thalamus ( $p=0.029$ ) (Figure 2). The ipsilateral rCBF on the symptomatic side was predominant in the parietal region, and the contralateral rCBF was predominant in the thalamus. The relationship between the $\mathrm{R} /(\mathrm{R}+\mathrm{L})$ ratios of the thalamus and parietal region was analyzed using Spearman correlation coefficient. However, the correlation analysis was not significant $(r s=-0.058, p=0.71)$.

With regard to the behavioral features (blaming dentists, sticking to dental treatment, and drug compliance), no significant differences were detected, except in the central region $(p=0.019)$, between the presence and absence of "sticking to dental treatment."

\section{Discussion}

The major findings of this study were as follows: (1) the patients who blamed their former dentists for inadequate 


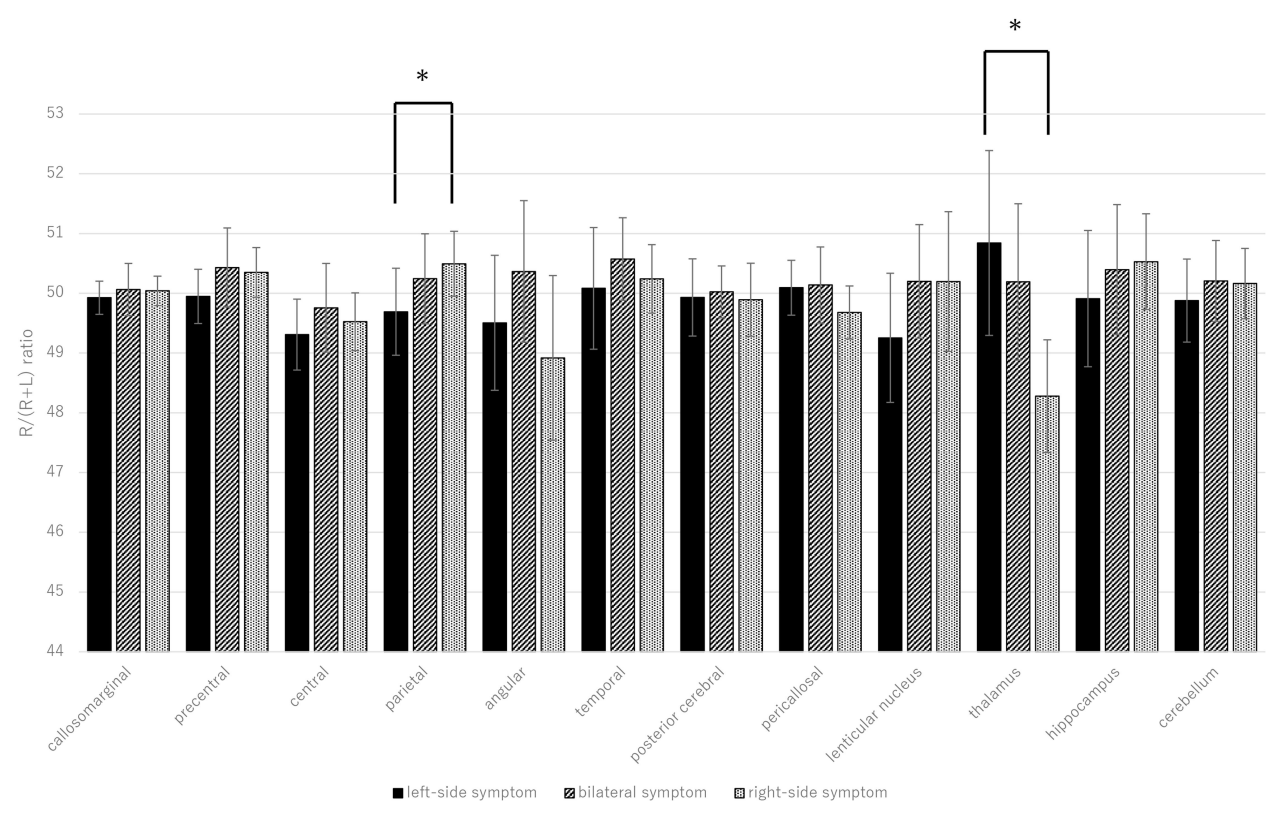

Figure $2 \mathrm{R} /(\mathrm{R}+\mathrm{L})$ ratios for each brain area in the three groups of patients with phantom bite syndrome (PBS) classified according to the symptomatic side (left, bilateral, and right). The $\mathrm{X}$-axis shows each region, and the $\mathrm{Y}$-axis shows the $\mathrm{R} /(\mathrm{R}+\mathrm{L})$ ratio. The graphs on the left (black bars), middle (hatched bars), and right (dotted bars) show the mean \pm SD for the patients with left-side symptoms, patients with bilateral symptoms, and patients with right-side symptoms, respectively. Kruskal-Wallis test and MannWhitney $U$-test corrected with Scheffe's test were used to compare the $R /(R+L)$ ratios for various areas of the brain of patients with PBS. There were significant differences between the patients with left- and right-side symptoms in the parietal region and thalamus $(* p<0.05)$.

dental treatments tended to have a symmetrical rCBF pattern on qualitative evaluation, and (2) rCBF asymmetry was detected quantitatively, corresponding only to the side of occlusal discomfort among the various clinical features. Patients with right-sided symptoms showed right-side-predominant $\mathrm{rCBF}$ asymmetry in the parietal region and leftside-predominant rCBF asymmetry in the thalamus, and vice versa. This study demonstrated the diversity of asymmetrical cerebral blood flow patterns corresponding to the clinical features of PBS.

The physiological oral sensation is mainly projected to the opposite thalamus and primary sensory cortex, and partly to the thalamus and primary sensory area of the ipsilateral side. $^{22,23}$ In this study, the patients with rightside symptoms were left-dominant in the thalamus and the patients with left-side symptoms were right-dominant in the thalamus, but the reason behind this is difficult to discern. Correlation analysis between the thalamus and parietal region was not significant, suggesting that the relationship between the asymmetry in the cerebral cortex and thalamus to be complicated.

Patients with PBS feel that their occlusion is uncomfortable and repeatedly attempt to confirm that it is in place, although they appear to be normal (ie, no movement or behavioral abnormality). Considering such clinical symptoms, the simplest possibility is that the opposite thalamus may be more active as the main conduction pathway as the result of concentrating on the sense of one side of the oral cavity, although it is uncertain whether they are primary or secondary. ${ }^{24,25}$

There was significant parietal asymmetry on the symptomatic side. In contrast, the asymmetry in the thalamus was left-predominant in patients with right-sided symptoms and right-predominant in patients with left-sided symptoms.

Previous studies have reported that masticatory muscle activity is positively and negatively correlated with cortical activation, including the frontal lobe. ${ }^{26}$ In a study that recorded 15 healthy subjects using fMRI, cortical activations in the primary sensorimotor cortex and cerebellum were positively correlated with masticatory muscle activity during molar biting. On the other hand, incisal biting was negatively correlated with the activity in the rostral cingulate motor area, superior frontal gyrus, and caudate nucleus. Moreover, experimentally reproduced occlusal discomfort in healthy participants changed the blood flow of the dorsolateral prefrontal cortex (DLPFC) and frontopolar cortex (FPC) in a near-infrared spectroscopy study ${ }^{27}$ and in the primary sensory cortex and Brodmann's area 46 in a fMRI study. ${ }^{28}$ DLPFC, FPC, and Brodmann's area 46 overlap with the precentral region in 3DSRT. Our results showed that significant asymmetry in the precentral region 
was detected only by Kruskal-Wallis test, not by Scheffe's multiple comparison. Experimentally reproduced occlusal discomfort and pathological PBS symptoms are certainly not identical, but it is interesting that the asymmetrical rCBF tendency was observed in the same area. Further studies confirming the relationship between the frontal area and PBS will be required.

The parietal area in 3DSRT has not been given much attention during the physiological experiments on occlusion. The parietal area includes the secondary sensory cortex,${ }^{20,21}$ which is considered to participate in the integration of various sensations. ${ }^{29,30}$ Considering the pathophysiology of PBS, it is not surprising that patients feel sustained occlusal discomfort if an abnormality in the secondary sensory cortex fails to integrate various sensations related to occlusion. ${ }^{25}$

The quantitative analysis in this study showed a tendency for contralateral rCBF increase in the thalamus in patients with PBS with right- and left-sided symptoms, suggesting some perfusion imbalance. However, no asymmetry in $\mathrm{rCBF}$ was found in patients with bilateral symptoms $(n=24 / 44)$, whose distribution was similar to that in the control group of our previous case-control study. ${ }^{17}$ Thus, some patients with PBS showed apparent rCBF asymmetry, but others did not. In fact, the visual assessment classified all 44 cases into 17 symmetrical and 27 asymmetrical cases, suggesting that more than half the patients had an asymmetrical rCBF pattern. Interestingly, the contingency table analysis showed a significant difference between symmetry in SPECT imaging and "blaming dentists."

In the clinical dental setting, patients with PBS are probably the most difficult to treat; ${ }^{7,31-34}$ they tend to complain about their dentists more directly. Sometimes, they even blame dentists for ineffective treatments ${ }^{31,35}$, The difficulty in maintaining a good dentist-patient relationship may lead to treatment dropout. ${ }^{6,14,36}$ Although patients regularly visit the dentist, they are skeptical about explaining the disease and/or treatment effectiveness, which may result in rejection or discontinuation of medication. ${ }^{14}$

Furthermore, the aggressive attitude of patients with PBS may be one of the main problems; however, our results demonstrated that patients who blame dentists tended to have a symmetrical rCBF pattern, which was unexpected. Although the interpretation of this result is not feasible, it was speculated that at least two subgroups might be included in patients with PBS. Patients in the first subgroup may have an asymmetrical rCBF pattern that might be related to an abnormality or dysfunction in their sensory system that generates misinterpretation of their occlusal status (similar to illusion or hallucination), causing phantom bite symptoms. On the other hand, the cerebral blood flow imbalance in patients of the second subgroup was not noticeable; the issue with the sensory system did not seem to be dominant. Some psychological factors such as feeling of being victimized, rather than sensory system dysfunction, might appear as the reason for their occlusal discomfort complaint, which was reported by Marbach ${ }^{2}$ and Jagger et al. ${ }^{6}$ Our previous case report, ${ }^{16}$ which presented an asymmetrical perfusion pattern that disappeared after effective treatment, might be classified into the first subgroup. However, a more detailed examination of the relationship between $\mathrm{rCBF}$ and psychological factors is necessary for adequate treatment.

In a recent review, ${ }^{37}$ various treatment options were suggested. In our clinic, pharmacotherapy using antidepressants and antipsychotic drugs was carried out based on previous studies. ${ }^{10,16}$ Pharmacotherapy might affect the balance of rCBF in PBS patients, ${ }^{16}$ however, it is often difficult for the patients to understand the necessity of psychotropic medications. As a result, most of the patients (especially in the second subgroup) stick to the dental procedures and resist taking medication. Our results might help these patients to understand their situation and increase the chance to accept pharmacotherapy apart from repeated dental procedures.

This study has several limitations. Methodologically, we should have performed correction for PVEs because they could underestimate rCBF values calculated by 3DRST in small structures of the brain. ${ }^{38}$ We could not perform PVE correction in our study because of the lack of brain MRI suitable for voxel-based morphometry (VBM). Further studies including both brain perfusion SPECT and MRI-based VBM are needed. Some patients with PBS had a psychiatric history and were on low-dose medications such as antidepressants and antipsychotics, which might have changed their $\mathrm{rCBF}$ patterns (a major limitation of the present study). Twenty-six of 44 patients had a psychiatric history such as depression $(n=13,29.5 \%)$, insomnia $(n=7,15.9 \%)$, adjustment disorder $(\mathrm{n}=2,4.5 \%)$, anxiety disorder $(\mathrm{n}=3$, $6.8 \%)$, and somatoform disorder $(n=2,4.6 \%)$ (including multiple diagnoses). However, no significant differences in age and sex were detected between patients with PBS with and without psychiatric comorbidity using the MannWhitney $U$-test. Additionally, we reported that the effect of psychiatric history on the asymmetrical rCBF patterns in 
patients with PBS was not significant. ${ }^{17,19}$ Hence, the significance in the present study might not disappear after the exclusion of the patients with PBS having a psychiatric history. The small sample size is also a limitation; thus, more studies with a larger number of participants are necessary.

\section{Conclusions}

In conclusion, the analysis of symptom laterality revealed that the patients with PBS showed blood flow imbalance in the thalamus and parietal region. Besides, patients with PBS who had symmetrical rCBF patterns show a tendency to blame their former dentists. Taken together, there are PBS patients who have slight disturbances of $\mathrm{rCBF}$ and those have not; suggesting the pathophysiology in these two groups might be different. These findings are useful for dentists in re-orientating the management of PBS. Still, the treatment for PBS is challenging, functional brain imaging might help the patients to understand their situation and increase the chance to accept pharmacotherapy apart from repeated dental procedures.

\section{Abbreviations}

PBS, Phantom bite syndrome; SPECT, single-photon emission computed tomography; $\mathrm{rCBF}$, regional cerebral blood flow; ${ }^{99 \mathrm{~m}} \mathrm{Tc}-\mathrm{ECD},{ }^{99 \mathrm{~m}} \mathrm{Tc}-\mathrm{ethyl}$ cysteinate dimer; HDS-R, Revised Hasegawa Dementia Scale; MRI, magnetic resonance imaging; 3DSRT, Three-dimensional stereotactic regions of interest template; DLPFC, dorsolateral prefrontal cortex; FPC, frontopolar cortex; VBM, voxel-based morphometry; PVE, partial-volume effects.

\section{Data Sharing Statement}

The datasets used and/or analyzed during the current study are available from the corresponding author upon reasonable request.

\section{Ethics Approval and Consent to Participate}

This study was conducted with the approval of the Ethical Committee of Tokyo Medical and Dental University (No. $24,593,141)$. Written informed consent was obtained from all participants.

\section{Consent for Publication}

Written informed consent for publication was obtained from all participants.

\section{Acknowledgments}

This study was supported in part by JSPS KAKENHI, Grant Numbers 20K18654, 19K10328 and 18K17306. The authors have no competing interests to report concerning this study. This study was also partially funded by the MEXT-Supported Program for the Strategic Research Foundation at Private Universities (S1511018).

\section{Author Contributions}

YU and YSh participated in the study design, performed the statistical analysis, and drafted the manuscript; HM and MW conceived the study, participated in its design and coordination, and helped in drafting the manuscript. AM, SS, KK, TT, TW, TS, MT, TY, and TN participated in data collection. ATor and JO performed and assessed the SPECT and helped draft the manuscript. YSa and IM assessed the participants' occlusion as prosthodontic specialists. AToy contributed to conception, design, data acquisition, and interpretation, and drafted and critically revised the manuscript. All authors gave final approval and agreed to be accountable for all aspects of this work. All authors contributed to data analysis, drafting or revising the article, have agreed on the journal to which the article will be submitted, gave final approval of the version to be published, and agree to be accountable for all aspects of the work.

\section{Funding}

There is no funding to report.

\section{Disclosure}

The authors declare that they have no competing interests for this work.

\section{References}

1. Marbach JJ. Phantom bite. Am J Orthod. 1976;70(2):190-199. doi:10.1016/S0002-9416(76)90319-5

2. Marbach JJ. Phantom bite syndrome. Am J Psychiatry. 1978;135 (4):476-479. doi:10.1176/ajp.135.4.476

3. Marbach JJ, Varoscak JR, Blank RT, Lund P. "Phantom bite": classification and treatment. J Prosthet Dent. 1983;49(4):556-559. doi:10.1016/0022-3913(83)90322-0

4. Melis M, Zawawi KH. Occlusal dysesthesia: a topical narrative review. J Oral Rehabil. 2015;42(10):779-785. doi:10.1111/joor.12309

5. Reeves JL, Merrill RL. Diagnostic and treatment challenges in occlusal dysesthesia. J Calif Dent Assoc. 2007;35(3):198-207.

6. Jagger RG, Korszun A. Phantom bite revisited. Br Dent J. 2004;197 (5):241-243. doi:10.1038/sj.bdj.4811613

7. Tsukiyama Y, Yamada A, Kuwatsuru R, Koyano K. Bio-psycho-social assessment of occlusal dysaesthesia patients. J Oral Rehabil. 2012;39 (8):623-629. doi:10.1111/j.1365-2842.2012.02317.x 
8. Hara ES, Matsuka Y, Minakuchi H, Clark GT, Kuboki T. Occlusal dysesthesia: a qualitative systematic review of the epidemiology, aetiology and management. J Oral Rehabil. 2012;39(8):630-638. doi:10.1111/j.1365-2842.2012.02300.x

9. Leon-Salazar V, Morrow L, Schiffman EL. Pain and persistent occlusal awareness: what should dentists do? J Am Dent Assoc. 2012;143 (9):989-991. doi:10.14219/jada.archive.2012.0325

10. Toyofuku A, Kikuta T. Treatment of phantom bite syndrome with milnacipran ? A case series. Neuropsychiatr Dis Treat. 2006;2 (3):387-390. doi:10.2147/nedt.2006.2.3.387

11. Davis KD, Kiss ZHT, Luo L, Tasker RR, Lozano AM, Dostrovsky JO. Phantom sensations generated by thalamic microstimulation. Nature. 1998;391(6665):385-387. doi:10.1038/34905

12. Hanyu-Deutmeyer AA, Varacallo M Phantom limb pain; 2019. Available from: http://www.ncbi.nlm.nih.gov/pubmed/28846343. Accessed June 27, 2019.

13. Marbach JJ. Orofacial phantom pain: theory and phenomenology. $J$ Am Dent Assoc. 1996;127(2):221-229. doi:10.14219/jada. archive. 1996.0172

14. Watanabe M, Umezaki Y, Suzuki S, et al. Psychiatric comorbidities and psychopharmacological outcomes of phantom bite syndrome. $J$ Psychosom Res. 2015;78(3):255-259. doi:10.1016/j.jpsychores. 2014.11.010

15. Shinohara Y, Umezaki Y, Minami I, et al. Comorbid depressive disorders and left-side dominant occlusal discomfort in patients with phantom bite syndrome (PBS). J Oral Rehabil. 2019; joor.12872. doi:10.1111/joor.12872.

16. Umezaki Y, Tu TTH, Toriihara A, Sato Y, Naito T, Toyofuku A. Change of cerebral blood flow after a successful pharmacological treatment of phantom bite syndrome. Clin Neuropharmacol. 2019;42 (2):49-51. doi:10.1097/WNF.0000000000000328

17. Shinohara Y, Umezaki Y, Watanabe M, et al. Brain perfusion-based analysis of "phantom bite syndrome.". Jap J Psychosom Dent. 2018;33(2):75-81.

18. Umezaki Y, Katagiri A, Watanabe M, et al. Brain perfusion asymmetry in patients with oral somatic delusions. Eur Arch Psychiatry Clin Neurosci. 2013;263(4):315-323. doi:10.1007/s00406-013-03907

19. Watanabe M, Umezaki Y, Miura A, et al. Comparison of cerebral blood flow in oral somatic delusion in patients with and without a history of depression: a comparative case series. BMC Psychiatry. 2015;15:42. doi:10.1186/s12888-015-0422-0

20. Kobayashi S, Tateno M, Utsumi K, et al. Quantitative analysis of brain perfusion SPECT in Alzheimer's disease using a fully automated regional cerebral blood flow quantification software, 3DSRT. $J$ Neurol Sci. 2008;264(1-2):27-33. doi:10.1016/j.jns.2007.07.015

21. Takeuchi R, Matsuda H, Yoshioka K, Yonekura Y. Cerebral blood flow SPET in transient global amnesia with automated ROI analysis by 3DSRT. Eur J Nucl Med Mol Imaging. 2004;31(4):578-589. doi:10.1007/s00259-003-1406-8

22. Steindler DA. Trigeminocerebellar, trigeminotectal, and trigeminothalamic projections: a double retrograde axonal tracing study in the mouse. J Comp Neurol. 1985;237(2):155-175. doi:10.1002/ cne. 902370203

Neuropsychiatric Disease and Treatment

\section{Publish your work in this journal}

Neuropsychiatric Disease and Treatment is an international, peerreviewed journal of clinical therapeutics and pharmacology focusing on concise rapid reporting of clinical or pre-clinical studies on a range of neuropsychiatric and neurological disorders. This journal is indexed on PubMed Central, the 'PsycINFO' database and CAS, and
23. Tamura Y, Shibukawa Y, Shintani M, Kaneko Y, Ichinohe T. Oral structure representation in human somatosensory cortex. Neuroimage. $\quad 2008 ; 43(1): 128-135 . \quad$ doi:10.1016/j.neuroimage. 2008.06.040

24. Avivi-Arber L, Lee J-C, Sessle BJ. Dental occlusal changes induce motor cortex neuroplasticity. J Dent Res. 2015;94(12):1757-1764. doi: $10.1177 / 0022034515602478$

25. Lickteig R, Lotze M, Kordass B. Successful therapy for temporomandibular pain alters anterior insula and cerebellar representations of occlusion. Cephalalgia. 2013;33(15):1248-1257. doi:10.1177/ 0333102413491028

26. Yoshizawa H, Miyamoto JJ, Hanakawa T, Shitara H, Honda M, Moriyama K. Reciprocal cortical activation patterns during incisal and molar biting correlated with bite force levels: an fMRI study. Sci Rep. 2019;9(1):8419. doi:10.1038/s41598-019-44846-4

27. Ono Y, Kobayashi G, Hayama R, et al. Prefrontal hemodynamic changes associated with subjective sense of occlusal discomfort. Biomed Res Int. 2015;2015:1-10. doi:10.1155/2015/395705

28. Oda M, Yoshino K, Tanaka T, et al. Identification and adjustment of experimental occlusal interference using functional magnetic resonance imaging. BMC Oral Health. 2014;14(1):124. doi:10.1186/ 1472-6831-14-124

29. Wang J, Yang Y, Fan L, et al. Convergent functional architecture of the superior parietal lobule unraveled with multimodal neuroimaging approaches. Hum Brain Mapp. 2015;36(1):238-257. doi:10.1002/ $\mathrm{hbm} .22626$

30. Zhang S, Li C-SR. Functional clustering of the human inferior parietal lobule by whole-brain connectivity mapping of resting-state functional magnetic resonance imaging signals. Brain Connect. 2014;4(1):140130070445002. doi:10.1089/brain.2013.0191

31. Kelleher M, Rasaratnam L, Djemal S. The paradoxes of phantom bite syndrome or occlusal dysaesthesia ('Dysesthesia '). Dent Update. 2017;44(1):8-32. doi:10.12968/denu.2017.44.1.8

32. Ligas BB, Galang MTS, BeGole EA, Evans CA, Klasser GD, Greene CS. Phantom bite: a survey of US orthodontists. Orthodontics (Chic). 2011;12(1):38-47.

33. Toyofuku A. Psychosomatic problems in dentistry. Biopsychosocial Med. 2016;10(1):14. doi:10.1186/s13030-016-0068-2

34. Wong MTH, Tsang ACC. Phantom bite in a Chinese lady. $J$ Hong Kong Med Assoc. 1991;43(4):105-107.

35. Tinastepe N, Kucuk BB, Oral K. Phantom bite: a case report and literature review. CRANIO ${ }^{\circledR}$. 2015;33(3):228-231. doi:10.1179/ 2151090314Y.0000000002

36. Kato T, Umezaki Y, Naito T, Bourgeois D. Effects of dropping out of dental treatment on the oral health-related quality of life among middle-aged subjects using web research. PLoS One. 2018;13(10): e0205462. doi:10.1371/journal.pone. 0205462

37. Imhoff B, Ahlers MO, Hugger A, et al. Occlusal dysesthesia-A clinical guideline. $J$ Oral Rehabil. 2020;47(5):651-658. doi:10.1111/joor.12950

38. Matsuda H, Ohnishi T, Asada T, et al. Correction for partial-volume effects on brain perfusion SPECT in healthy men. $J$ Nucl Med. 2003;44(8):1243-1252. tely online and includes a very quick and fair peer-review system, which is all easy to use. Visit http://www.dovepress.com/testimonials.php to read real quotes from published authors.

\section{Dovepress}

